\title{
Study of the Mott Transition in the Three-Dimensional Hubbard Model
}

\author{
F. Mancini* AND V. Turkowski ${ }^{\dagger}$ \\ Dipartimento di Fisica "E.R. Caianiello" — Unità INFM di Salerno \\ Università degli Studi di Salerno, 84081 Baronissi (SA), Italy
}

(Received April 6, 2001; in final form December 20, 2001)

The Mott-Hubbard transition in the three-dimensional Hubbard model is studied by means of the composite operator method. Analytical and numerical calculations show the existence of a critical value $U_{c}$ of the Coulomb interaction which separates paramagnetic insulating and metallic phases. In the composite operator method the ratio $U_{c} / W$, where $W$ is the band width, has the same value as in the two-dimensional case. The ground state of the Mott insulator is characterized by a local antiferromagnetic order where the electrons keep some mobility, but this latter must be compatible with the local ordering. By analyzing the intersite hopping it is possible to establish that some correlation functions behave as order parameters in the insulating phase.

PACS numbers: 71.10.--w, 71.10.Fd, 71.30.+h

\section{Introduction}

The study of strongly correlated electron systems has a long history. Probably, the beginning of these studies can be traced back to a conference on electrical conduction mechanisms, held in Bristol in 1937 [1], where de Boer and Verwey presented [2] conductivity data for some transition metal oxides. Almost all materials demonstrated insulating behavior at room temperatures. However, according to Bloch theory they should be metals, because a simple account of the number of $3 d$ electrons gives a partially filled $3 d$ bands. The authors proposed that the

* corresponding author; e-mail: mancini@sa.infn.it

${ }^{\dagger}$ Present address: CFIF, Instituto Superior Tecnico, Av. Rovisco Pais, 1049-001 Lisbon, Portugal. 
Coulomb repulsion between $3 d$ electrons might be responsible for barrier for tunnelling between neighbor sites. In the following discussion Peierls proposed that the Coulomb repulsion leads to the particle localization: "it is quite possible that the electrostatic interaction between electrons prevents them from moving at all". Therefore, the Bloch wave theory could not describe the behavior of the materials and it was necessary to find another proper model. Then, beginning from 1949, Mott in a series of works [3] summarized the ideas proposed and suggested a new vision of the problem, which is known now as the problem of the Mott insulator. He estimated that the insulating gap in the electron spectra is of the order of the Coulomb repulsion integral on the same site. Therefore, it became more evident that the strong electron correlations are crucial in these phenomena. Beginning from that time the theoretical physicists started to model and investigate the behavior of strongly correlated electronic systems. The simplest effective model which takes into account these correlations is the Hubbard model (HM) [4]. Its Hamiltonian consists of a kinetic term, describing the inter-site hopping of electrons, and a potential term describing the intra-site Coulomb repulsion. In the early years the Hubbard model investigations were devoted mainly to the study of the Mott transition in vanadium and titanium oxides (the results for $\mathrm{V}_{2} \mathrm{O}_{3}$ are summarized in [5]). From the first works there has been an extensive study of this model (for overview see, for example, Refs. [6] and [7]). However, after more than thirty years a real full understanding of its properties is still lacking. The model has two exact solvable limits, $U=0$, when the Coulomb interaction is absent, and $t=0$, the atomic limit, when the inter-site hopping is absent. Unfortunately, the solution in the intermediate case of finite $t$ and $U$ is still lacking. In the one-dimensional (1D) case the Hubbard model is exactly integrable by means of the Bethe ansatz (BA) [8] and the ground state wave function is known [9]. In this way many properties are known exactly within the numerics needed in the case of arbitrary particle density and finite temperature. However, the BA does not provide a complete framework for describing the physics of the 1D Hubbard model since many properties, like the correlation and spectral functions, cannot be evaluated from the BA wave function except for some limiting cases.

Despite the absence of really satisfactory treatments, the Hubbard model is one of the most important models of the condensed matter physics today. The model can describe not only a metal-insulator transition [4, 10, 11], but also antiferromagnetism [12], ferromagnetism [13], superconductivity [14], and many other phenomena.

The Hubbard model investigations have received a new impulse after the discovery of high-temperature superconductors (HTSC). Superconducting oxides, such as $\mathrm{La}_{2-x} \mathrm{Sr}_{x} \mathrm{CuO}_{4}, \mathrm{Nd}_{2-x} \mathrm{Ce}_{x} \mathrm{CuO}_{4}, \mathrm{YBa}_{2} \mathrm{Cu}_{3} \mathrm{O}_{7-y}$ and $\mathrm{Bi}_{2} \mathrm{Sr}_{2} \mathrm{CaCu}_{2} \mathrm{O}_{8+\delta}$, have a layered structure of hole dynamics and in the underdoped regime are in an antiferromagnetic insulating state. Due to this, studies concerning HTSC have been carried out mainly in two-dimensional models. Many approaches have been 
proposed to study the 2D Hubbard model. Among them we cite the slave boson method [15], the $d_{\infty}$ method [16, 17], the projection operator method [18], the composite operator method (COM) [19a-19d]. There are many evidences now that this model can describe some of the HTSC properties. For example, it was shown [20] by using the COM that this model can qualitatively describe the behavior of several properties of cuprate compounds in the normal state.

Relatively not much attention has been paid to the study of the three-dimensional (3D) Hubbard model in the last years. However, there are several reasons for study such a model. First, there are some oxides with 3D strong electronic correlation structure, for example $\mathrm{YTiO}_{3}, \mathrm{SrVO}_{3}, \mathrm{Y}_{1-x} \mathrm{Ca}_{x} \mathrm{TiO}_{3}, \mathrm{Sr}_{1-x} \mathrm{La}_{x} \mathrm{TiO}_{3}$ and $\mathrm{La}_{2} \mathrm{CuO}_{4+\delta}$, which have been experimentally investigated in the last years (see, for instance, [21-29] and for an overview [7]). The possibility of describing photoemission results for $\mathrm{YTiO}_{3}$ and some other materials [21-23] has been also considered. In some perovskite oxide with $3 d^{1}$ configuration ( $\mathrm{Ti}^{3+}$, for example) the hopping amplitude $t$ can be changed by varying $d-d$ neighboring overlaps due to a tetragonal distortion. This means that the changing of composition leads to varying the ratio $U / t$. The Mott transition has been observed in the series $\mathrm{SrVO}_{3}$, $\mathrm{CaVO}_{3}, \mathrm{LaTiO}_{3}, \mathrm{YTiO}_{3}$. At small $U / t$ and half-filling, near $E_{\mathrm{F}}$ a quasiparticle and a high-energy band, the latter corresponding to the lower Hubbard band (LHB), were observed. Then, the spectral weight is redistributed from the quasiparticle band to the LHB by increasing $U / t$ (see $[17,21,30]$ ). Also, the metal-insulator transition driven by the band filling changing due to varying the chemical composition has been observed [23, 26, 31]. Thus, $\mathrm{Y}_{1-x} \mathrm{Ca}_{x} \mathrm{TiO}_{3}$ passes from the antiferromagnetic insulating to metallic state as $x$ increases. Two Hubbard subbands are visible in a wide range of $x$. (The theoretical description of this phenomena has been performed in [32] (see also $[17,33]$ ) and [30] in the $D=\infty$ and $D=3$ Hubbard model.)

Second, the study of the 3D Hubbard model may be useful for understanding the physics of the HTSC which are layered strongly correlated materials, i.e. they exhibit a behavior intermediate between $2 \mathrm{D}$ and $3 \mathrm{D}$ structure.

Let us mention some of the works devoted to the 3D Hubbard model which have appeared during the last 10-15 years. Spectral properties of the model were studied in [30] by using the Monte Carlo method, and in [34] and [35], in the weak-interaction limit, by using the $U$-expansion. One-electron energy was calculated in [36] by using an atomic basis with Wannier functions. Recently, the possibility of the Mott transition in the 3D Hubbard model was studied in [37] as a limiting case of the many-plane HM. The spectral and magnetic properties of the model were studied in [38] by using the fluctuation-exchange and the two-particle self-consistent approximation in the weak-coupling regime. Magnetic properties of the model were studied numerically in [39-41] and in [42, 43] along with thermodynamic ones in order to investigate the possibility of Néel transition. The authors of the papers $[44,45]$ mapped spin excitations on those of the effective underlying 
Heisenberg model to find the antiferromagnetic critical Néel temperature and to describe the magnetism in the 3D Hubbard model. The magnetic phase diagram was studied at second order in [46]. Magnetic properties in the 3D Hubbard model at half-filling were also studied in [47] by using the linked-cluster series expansion method. In a series of papers [48-51] the magnetic and thermodynamical properties of the model were studied by using the Gutzwiller variational approach and the single-site spin fluctuation theory. The ground state energy was calculated analytically in [52] at small $U$ at second order. The phase diagram of the 3D Hubbard model was studied in [53].

In this article we consider the paramagnetic solution of the three-dimensional Hubbard model by means of the composite operator method [19a-19d]. The motivation is to study the Mott-Hubbard transition and the nature of the ground state; therefore, we concentrate the analysis to half-filling and to zero temperature. By studying the chemical potential and the density of states we find that there exists a critical value $U_{\mathrm{c}}$ of the Coulomb potential which separates a paramagnetic metal for $U<U_{\mathrm{c}}$ and a Mott-Hubbard insulator for $U>U_{\mathrm{c}}$. This critical potential is determined by a self-consistent equation and is mainly controlled by the intersite fluctuations. We can show that at zero temperature the double occupancy remains finite in the insulating phase and tends asymptotically to zero as $U \rightarrow \infty$. The result that in the insulating phase there exists a small fraction of doubly occupied and empty sites is also confirmed by our study of the density of states. By decomposing the electron operator as $c=\xi+\eta$, where $\xi$ and $\eta$ are the usual Hubbard operators describing the transitions $(n=0) \Leftrightarrow(n=1)$ and $(n=1) \Leftrightarrow(n=2)$, respectively, we have studied the contribution of $\xi$ and $\eta$ to the density of states. As many analytical and numerical results also show, in the insulating phase the electron density of states is split into two bands, the lower and upper Hubbard bands, but there is always a contribution of $\xi(\eta)$ to the upper (lower) band. Furthermore, the cross term $\left\langle\xi \eta^{\dagger}\right\rangle$ contributes to the density of states in a significant way around the Fermi level, even for large values of $U$.

These results open questions about the nature of the ground state and the identification of an order parameter. To answer these questions we have studied the quantity $\left\langle c(i) c^{\dagger}(j)\right\rangle$, which gives the probability amplitude of hopping from site $i$ to site $j$. By means of the decomposition $c=\xi+\eta$ we are able to study how the latter quantity depends on the average occupation of the sites $i$ and $j$. We have investigated up to the third nearest neighbors, but the analysis can be easily extended to any distance, by symmetry considerations. The picture that emerges by this study is that an antiferromagnetic order is established in the insulating phase, but the situation is not the one as in the Heisenberg antiferromagnet where all electrons are frozen on the sites. The ground state is characterized by a small fraction, depending on the value of $U$, of empty and doubly occupied sites; the electrons can hop among sites, but there are strong constraints on their mobility such that the antiferromagnetic local order is not destroyed. The probability 
amplitudes $\left\langle\xi(i) \xi^{\dagger}\left(j_{\text {odd }}\right)\right\rangle$ and $\left\langle\eta(i) \eta^{\dagger}\left(j_{\text {odd }}\right)\right\rangle$, where $j_{\text {odd }}$ is an odd nearest neighboring site of $i$, vanish at $U=U_{c}$ and remain zero for all $U>U_{c}$, putting a constraint on the electron mobility. We might take these quantities as the ones that characterize the order in the insulating phase. This picture is consistent with the fact that there is a competition between the itinerant and localizing energy terms; for any finite value of $t$ there is always a small contribution coming from kinetic energy which allows the hopping among sites. However, at a certain value of $U$ a local magnetic order is established and the hopping is severely restricted. Only in the limit of infinite $U$ the electron mobility is totally forbidden and the system becomes a Heisenberg antiferromagnet.

In Sec. 2, by means of the technique of the equations of motion, we derive the explicit expression for the retarded Green function (GF) in the two-pole approximation. The GF depends on some parameters that must be self-consistently calculated. By requiring that the representation of the GF satisfies symmetry constraints, a set of self-consistent equations is derived. By solving these equations in Sec. 3 we study the problem of the Mott transition. The existence of metal-insulator transition is investigated by looking at the chemical potential, the density of states and the probability amplitudes $\left\langle c(i) c^{\dagger}(j)\right\rangle$. Some conclusions are drawn in Sec. 4. Details of calculations are reported in Appendix.

\section{Formulation}

The Hubbard model is described by the following Hamiltonian:

$$
H=H_{t}+H_{U} .
$$

$H_{t}$ is the kinetic term which describes the motion of the electrons among the sites of a Bravais lattice spanned by the vectors $i$ :

$$
H_{t}=\sum_{i, j}\left(t_{i j}-\mu \delta_{i j}\right) c^{\dagger}(i) c(j) .
$$

$c(i), c^{\dagger}(i)$ are annihilation and creation operators of electrons at site $i=(i, t)$ in the spinor notation

$$
c=\left(\begin{array}{c}
c_{\uparrow} \\
c_{\downarrow}
\end{array}\right), \quad c^{\dagger}=\left(\begin{array}{cc}
c_{\uparrow}^{\dagger} & c_{\downarrow}^{\dagger}
\end{array}\right)
$$

and satisfy canonical anticommutation relations

$$
\begin{aligned}
& \left\{c_{\sigma}(\boldsymbol{i}, t), c_{\sigma^{\prime}}^{\dagger}(\boldsymbol{j}, t)\right\}=\delta_{\sigma, \sigma^{\prime}} \delta_{\boldsymbol{i j}}, \\
& \left\{c_{\sigma}(\boldsymbol{i}, t), c_{\sigma^{\prime}}(\boldsymbol{j}, t)\right\}=\left\{c_{\sigma}^{\dagger}(\boldsymbol{i}, t), c_{\sigma^{\prime}}^{\dagger}(\boldsymbol{j}, t)\right\}=0,
\end{aligned}
$$

$\mu$ is the chemical potential, $t_{i j}$ denotes the transfer integral between different sites. We fix the scale of energy in such a way that $t_{\boldsymbol{i} i}=0$. In addition to the band energy the model contains an interaction term which approximates the correlation among the electrons. In the simplest form the interaction is between electrons of opposite 
spin on the same lattice site; the strength of the interaction is described by the parameter $U$ :

$$
H_{U}=U \sum_{i} n_{\uparrow}(i) n_{\downarrow}(i)
$$

where $n_{\sigma}(i)=c_{\sigma}^{\dagger}(i) c_{\sigma}(i)$ is the number operator for the electrons with spin $\sigma$. In the nearest-neighbor approximation the hopping matrix for a $d$-dimensional hyper cubic lattice with lattice constant $a$ takes the form $t_{i j}=-2 d t \alpha_{i j}$, where $\alpha_{i j}$ is the projector on the nearest neighbor sites

$$
\alpha_{i \boldsymbol{j}}=\frac{1}{N} \sum_{\boldsymbol{k}} \mathrm{e}^{\mathrm{i} \boldsymbol{k}(\boldsymbol{i}-\boldsymbol{j})} \alpha(\boldsymbol{k}), \quad \alpha(\boldsymbol{k})=\frac{1}{d} \sum_{n=1}^{d} \cos \left(k_{n} a\right),
$$

$N$ is the number of the sites and the summation runs over the first Brillouin zone.

A convenient basis to study this model is given by the composite field

$$
\psi(i)=\left(\begin{array}{l}
\xi(i) \\
\eta(i)
\end{array}\right)
$$

where $\xi(i)$ and $\eta(i)$ are the Hubbard fields

$$
\xi(i)=[1-n(i)] c(i), \quad \eta(i)=n(i) c(i) .
$$

The field $\xi(i)$ is responsible for the transitions $|0\rangle \leftrightarrow|\sigma\rangle$, while $\eta(i)$ takes care of the transitions $|\sigma\rangle \leftrightarrow|\uparrow \downarrow\rangle .|0\rangle$ denotes the state with no $c$-particles. $n(i)=c^{\dagger}(i) c(i)$ is the total number operator. The Heisenberg field (2.7) satisfies the equation of motion

$$
\mathrm{i} \frac{\partial}{\partial t} \psi(i)=J(i)=\left(\begin{array}{c}
-\mu \xi(i)-2 d t\left[c^{\alpha}(i)+\pi(i)\right] \\
(U-\mu) \eta(i)+2 d t \pi(i)
\end{array}\right)
$$

where $\pi(i)$ is a higher-order composite field defined as

$$
\pi(i)=\frac{1}{2} \sigma^{\mu} n_{\mu}(i) c^{\alpha}(i)+c(i) c^{\alpha^{\dagger}}(i) c(i)
$$

We have introduced the charge $(\mu=0)$ and spin $(\mu=1,2,3)$ density operator $n_{\mu}(i)=c^{\dagger}(i) \sigma_{\mu} c(i) . \sigma_{\mu}$ are the Pauli matrices, with the notation $\sigma_{\mu}=(1, \boldsymbol{\sigma})$ and $\sigma^{\mu}=(-1, \boldsymbol{\sigma})$. For any operator $\Phi$, the notation $\Phi^{\alpha}(i)$ stands for

$$
\Phi^{\alpha}(i)=\sum_{j} \alpha_{i} j^{\Phi(j)}
$$

In order to solve the Heisenberg equation (2.9) we project the source $J(i)$ on the basic field

$$
J(i)=\sum_{j} \varepsilon(i, j) \psi(j) .
$$

In the weak sense the matrix $\varepsilon(\boldsymbol{i}, \boldsymbol{j})$ is determined by the following equation:

$$
\left\langle\left\{J(i, t), \psi^{\dagger}(l, t)\right\}\right\rangle=\sum_{j} \varepsilon(i, j)\left\langle\left\{\psi(j, t), \psi^{\dagger}(l, t)\right\}\right\rangle .
$$


The notation $\langle\cdots\rangle$ stands for the quantum statistical average in the grand canonical ensemble.

Let us consider the thermal retarded Green function

$$
S(i, j)=\left\langle R\left[\psi(i) \psi^{\dagger}(j)\right]\right\rangle=\theta\left(t_{i}-t_{j}\right)\left\langle\left\{\psi(i), \psi^{\dagger}(j)\right\}\right\rangle .
$$

By means of (2.12) the Fourier transform of $S(i, j)$ satisfies the equation

$$
[\omega-\varepsilon(k)] S(k, \omega)=I(k),
$$

where $\varepsilon(k)$ is the Fourier transform of the energy matrix $\varepsilon(i, j)$ and $I(k)$ is the normalization matrix

$$
I(\boldsymbol{k})=\mathrm{F} . \mathrm{T} .\left\langle\left\{\psi(\boldsymbol{i}, t), \psi^{\dagger}(\boldsymbol{j}, t)\right\}\right\rangle .
$$

The symbol F.T. denotes the Fourier transform. The solution of (2.15) is given by

$$
S(k, \omega)=\sum_{n=1}^{2} \frac{\sigma^{(n)}(k)}{\omega-E_{n}(k)+\mathrm{i} \delta},
$$

where $E_{n}(k)$ are the eigenvalues of the matrix $\varepsilon(k)$ and the spectral functions $\sigma^{(n)}(k)$ can be calculated by means of the equation

$$
\sigma_{a b}^{(n)}(k)=\Omega_{a n}(k) \sum_{c} \Omega_{n c}^{-1}(k) I_{c b}(k),
$$

where $\Omega(k)$ is the $2 \times 2$ matrix whose columns are the eigenvectors of the matrix $\varepsilon(k)$. By considering a paramagnetic homogeneous state, straightforward calculations give the following results. The energy spectra $E_{n}(k)$ and the spectral functions are given by

$$
\begin{aligned}
& E_{1}(k)=R(k)+Q(k), \quad E_{2}(k)=R(k)-Q(k), \\
& \sigma_{11}^{(1)}(k)=\frac{I_{11}}{2}\left[1+\frac{g(k)}{2 Q(k)}\right], \quad \sigma_{11}^{(2)}(k)=\frac{I_{11}}{2}\left[1-\frac{g(k)}{2 Q(k)}\right], \\
& \sigma_{12}^{(1)}(k)=\frac{m_{12}(k)}{2 Q(k)}, \quad \sigma_{12}^{(2)}(k)=-\frac{m_{12}(k)}{2 Q(k)}, \\
& \sigma_{22}^{(1)}(k)=\frac{I_{22}}{2}\left[1-\frac{g(k)}{2 Q(k)}\right], \quad \sigma_{22}^{(2)}(k)=\frac{I_{22}}{2}\left[1+\frac{g(k)}{2 Q(k)}\right],
\end{aligned}
$$

where

$$
\begin{array}{r}
R(k)=-\mu-2 d t \alpha(k)+\frac{1}{2} U-\frac{m_{12}(k)}{2 I_{11} I_{22}}, \quad Q(k)=\frac{1}{2} \sqrt{g^{2}(k)+\frac{4 m_{12}^{2}(k)}{I_{11} I_{22}},} \\
g(k)=-U+\frac{1-n}{I_{11} I_{22}} m_{12}(k), \quad m_{12}(k)=2 d t\left[\Delta+\alpha(k)\left(p-I_{22}\right)\right] .
\end{array}
$$

$I_{a b}(k)$ are the elements of the normalization matrix

$$
I(k)=\left(\begin{array}{cc}
I_{11} & 0 \\
0 & I_{22}
\end{array}\right), \quad I_{11}=1-\frac{1}{2} n, \quad I_{22}=\frac{1}{2} n .
$$


$n=\left\langle c^{\dagger}(i) c(i)\right\rangle$ is the a verage particle number per site. The parameters $\Delta$ and $p$ describe a constant shift of the bands and a band width renormalization, respectively. They are static intersite correlation functions defined as

$$
\begin{aligned}
& \Delta \equiv\left\langle\xi^{\alpha}(i) \xi^{\dagger}(i)\right\rangle-\left\langle\eta^{\alpha}(i) \eta^{\dagger}(i)\right\rangle \\
& p \equiv \frac{1}{4}\left\langle n_{\mu}^{\alpha}(i) n_{\mu}(i)\right\rangle-\left\langle\left[c_{\uparrow}(i) c_{\downarrow}(i)\right]^{\alpha} c_{\downarrow}^{\dagger}(i) c_{\uparrow}^{\dagger}(i)\right\rangle .
\end{aligned}
$$

The correlation function $C(i, j)=\left\langle\psi(i) \psi^{\dagger}(j)\right\rangle$ can be calculated from (2.17) by means of the spectral theorem and has the expression

$C(i, j)=\frac{\Omega}{(2 \pi)^{d}} \sum_{n=1}^{2} \int_{\Omega_{\mathrm{B}}} \mathrm{d}^{d} k \mathrm{e}^{\mathrm{i} \boldsymbol{k} \cdot(\boldsymbol{i}-j)-i} E_{n}(\boldsymbol{k})\left(t_{i}-t_{j}\right)\left\{1-f\left[E_{n}(\boldsymbol{k})\right]\right\} \sigma^{(n)}(\boldsymbol{k})$.

$\Omega$ and $\Omega_{\mathrm{B}}$ are the volumes of the unit cell in the direct and reciprocal lattices, respectively. $f\left[E_{n}(\boldsymbol{k})\right]=\left[\mathrm{e}^{\beta E_{n}(\boldsymbol{k})}+1\right]^{-1}$ is the Fermi distribution function. The Green function $S(i, j)$ and the correlation function $C(i, j)$ depend on the internal parameters $\mu, \Delta, p$, which must be self-consistently determined. In the framework of the COM these parameters are fixed by the following set of coupled equations:

$$
\begin{aligned}
& n=2\left(1-C_{11}-C_{22}\right), \\
& \Delta=C_{11}^{\alpha}-C_{22}^{\alpha}, \\
& C_{12}=0,
\end{aligned}
$$

where we use the notation $C=\left\langle\psi(i) \psi^{\dagger}(i)\right\rangle, C^{\alpha}=\left\langle\psi^{\alpha}(i) \psi^{\dagger}(i)\right\rangle$.

As well known, the equations of motion are not sufficient to determine the Green function. Generally speaking, this quantity is defined as the quantum statistical average of some operators on a given vectorial space. The Hilbert space, where the operators act, must be defined. In the ordinary perturbation theory the Green function is expressed in terms of the free propagator and the Hilbert space is chosen as the Fock space of the free electron field. In this case Eq. (2.27) $n=\left\langle c^{\dagger}(i) c(i)\right\rangle$, which expresses the chemical potential in terms of the particle number and temperature, is completely sufficient to determine the representation of the Green function. In the presence of strong electronic correlations the ordinary perturbation theory completely fails and other techniques must be developed. One framework of calculations is based on the use of higher-order (composite) operators as basis for developing perturbation schemes. This approach is very convenient because some amount of the interaction is already contained in the chosen basis and permits to overcome the problem of finding an appropriate expansion parameter. However, one price must be paid. These composite fields are neither fermion nor boson fields; they satisfy a noncanonical algebra and their properties, because of its inherent definition, must be self-consistently determined. For example, the composite operator (2.7) satisfies the following algebra:

$$
\left\{\xi(i, t), \xi^{\dagger}(j, t)\right\}=\delta_{i j}\left[1+\frac{1}{2} \sigma^{\mu} n_{\mu}(i)\right]
$$




$$
\left\{\eta(i, t), \eta^{\dagger}(j, t)\right\}=-\frac{1}{2} \delta_{i j} \sigma^{\mu} n_{\mu}(i)
$$

As a result, the propagator $\left\langle R\left[\psi(i) \psi^{\dagger}(j)\right]\right\rangle$ depends on the parameters $\mu, \Delta, p$ which have to be determined. Several methods (decoupling schemes, use of the equations of motion) have been considered in the literature in the context of different approaches (Hubbard I approximation, Roth's method, projection method, spectral density approach). However, as shown in Ref. [19b] all these procedures lead to a series of unpleasant results: several sum rules and the particle-hole symmetry are violated, there is no Mott transition, all local quantities strongly disagree with the results of numerical simulations. The reason is that in those approaches no attention is paid to the problem of fixing the representation of the Green function. When the algebra is noncanonical the self-consistent Eq. (2.27) which fixes the chemical potential is not sufficient to completely determine the representation. The other parameters $\Delta, p$ remain to be fixed. The parameter $\Delta$, defined by (2.24), will be determined by the self-consistent Eq. (2.28). It is worthwhile to recall that in the Hubbard I approximation this parameter is put equal to zero; this procedure is completely inconsistent. The other parameter $p$, defined by $(2.25)$, is fixed by requiring that the Pauli principle

$$
\xi_{\sigma}(i) \eta_{\sigma}^{\dagger}(i)=c_{\sigma}(i)\left[n_{-\sigma}(i)-n_{-\sigma}^{2}(i)\right] c_{\sigma}^{\dagger}(i)=0
$$

be satisfied also at the level of matrix elements

$$
\left\langle\xi_{\sigma}(i) \eta_{\sigma}^{\dagger}(i)\right\rangle=0
$$

Straightforward calculations show that Eq. (2.32) is systematically violated unless the three parameters are fixed by the set of coupled Eqs. (2.27)-(2.29). We have shown [54] that this procedure of fixing the parameters $\mu, \Delta, p$ is exact in the case of a solvable model and gives very reasonable results for the one- and two-dimensional Hubbard model (see for example: [19a, 55]). In particular, the particle-hole symmetry is conserved, all the local quantities are in good agreement with the results of numerical simulations and with the Bethe-ansatz calculations.

In this article we want to study the metal-insulator transition in the case of a three-dimensional system. Therefore, we concentrate our attention to half-filling. In this regime the self-consistent Eqs. (2.27)-(2.29) give $\mu=U / 2$ and $\Delta=0$, in agreement with the general relations

$$
\mu(2-n)=U-\mu(n), \quad \Delta(2-n)=-\Delta(n)
$$

required by the particle-hole symmetry. In order to calculate the value of the parameter $p$ at half-filling, let us define

$$
-\mu_{1}=\left(\frac{\partial \mu}{\partial n}\right)_{n=1}=\frac{1}{\kappa(1)}, \quad \Delta_{1}=\left(\frac{\partial \Delta}{\partial n}\right)_{n=1},
$$

where $\kappa(n)$ is the compressibility $\kappa(n)=(\partial n / \partial \mu) / n^{2}$. Then, from $(2.27)-(2.29)$ it is possible to derive [56] the following system of equations: 


$$
\begin{aligned}
& 4 \mu_{1} K_{1}+16 d t\left[K_{1}-2 d t(2 p-1) K_{2}\right] \Delta_{1}=\left(2+U K_{3}\right)-4 d t(2 p-1) U K_{2}, \\
& 2 U K_{2} \mu_{1}+4\left[1+2 d t U K_{2}+2 U d^{2} t^{2}(2 p-1)\left(2 K_{4}-K_{6}\right)\right] \Delta_{1} \\
& \quad=2 K_{7}+4 d t(2 p-1) K_{5}+d t(2 p-1) U^{2}\left(2 K_{4}-K_{6}\right), \\
& 2(2 p-1) K_{2} \mu_{1}+2\left[K_{3}+4 d t(2 p-1) K_{2}+4 d^{2} t^{2}(2 p-1)^{2}\left(2 K_{4}-K_{6}\right)\right] \Delta_{1} \\
& \quad=d t(2 p-1)^{2} U\left(2 K_{4}-K_{6}\right),
\end{aligned}
$$

where the quantities $K_{i}(i=1,7)$, defined in Appendix, are expressed only in terms of $p$.

\section{The Mott-Hubbard transition}

By solving the system of self-consistent Eqs. (2.27)-(2.29), the chemical potential can be calculated as a function of the external parameters filling $n$, temperature $T$, and Coulomb potential $U$. In this article all the energies are measured in units of $t$, the transfer integral. Some of the results are shown in Fig. 1, where $\mu$ is plotted versus the particle density at $T=0$ and several values of $U$. As expected, $\mu$ takes the value $\mu=-6 t$ at $n=0$ and increases by increasing $n$. For large values of $U$ our results show a discontinuity of the chemical potential at half-filling, signalling the presence of a gap in the density of states. To better study this aspect, in Fig. 2 we give the quantity $\mu_{1}=-(\partial \mu / \partial n)_{n=1}$ as a function of the interaction strength for $T=0.0001$ and $T=1$. We see that at finite temperature $(\partial \mu / \partial n)_{n=1}$ increases by increasing $U$ and tends to diverge in the limit of $U \rightarrow \infty$. At zero temperature $\mu_{1}$ diverges at a critical value of $U: U_{c} \approx 1.68 \mathrm{~W}$, where $W=12 t$ is

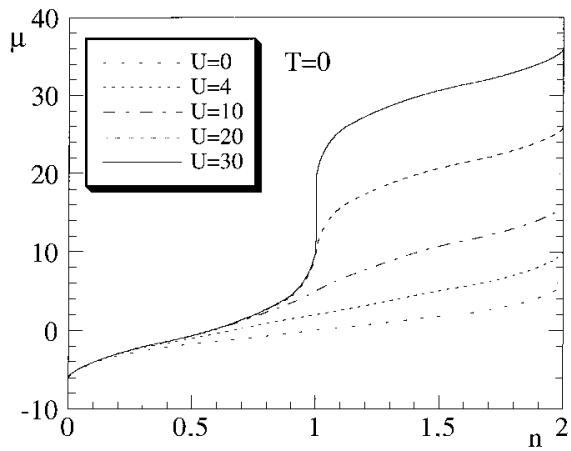

Fig. 1

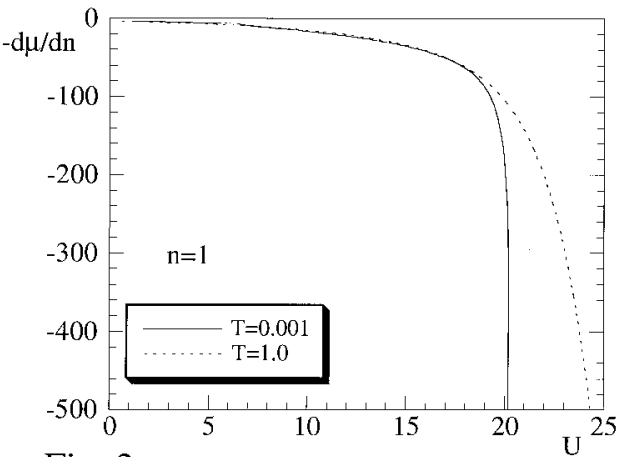

Fig. 2

Fig. 1. The chemical potential is plotted as a function of the particle density at zero temperature and several values of $U$.

Fig. 2. The parameter $\mu_{1}=-(\partial \mu / \partial n)_{n=1}$ is plotted as a function of the potential strength $U / t$ for $T=0.0001$ and $T=1$. 
the band width. At this value of $U$ there is a phase transition from metallic to insulating state.

The density of states (DOS) for the c-electrons is given by the following expression:

$$
\begin{aligned}
N(\omega) & =\frac{\Omega}{(2 \pi)^{3}} \sum_{\alpha, \beta=1}^{2} \int_{\Omega_{\mathrm{B}}} \mathrm{d}^{3} k\left(-\frac{1}{\pi}\right) \operatorname{Im}\left[S_{\alpha \beta}(\boldsymbol{k}, \omega)\right] \\
& =\frac{\Omega}{(2 \pi)^{3}} \sum_{\alpha, \beta=1}^{2} \sum_{n=1}^{2} \int_{\Omega_{\mathrm{B}}} \mathrm{d}^{3} k \delta\left[\omega-E_{n}(\boldsymbol{k})\right] \sigma_{\alpha \beta}^{(n)}(\boldsymbol{k}) .
\end{aligned}
$$

In Fig. 3 the DOS is plotted as a function of the energy $\omega$ at zero temperature, half-filling and several values of $U$. The figure shows characteristic van Hove singularities corresponding to saddle points in the energy spectra $E_{n}(\boldsymbol{k})$. Contrary to the $2 \mathrm{D}$ case, the DOS have a plateau, i.e. many-quasiparticle resonances, around the Fermi level at small $U$. The same result has been obtained in Refs. [15] and [36]. However, the peak obtained in these papers is rather large at small $U$. By increasing $U$ the central peak opens in two peaks: some of the central weight is transferred to the two peaks that correspond to the elementary excitations described by the fields $\xi$ and $\eta$. For small $U$ the two bands overlap and the system is in the metallic state. The overlapping region is given by

$$
\Delta \omega=E_{1}(\mathbf{0})-E_{2}(\boldsymbol{Q})=-24 t p+\sqrt{U^{2}+[12 t(2 p-1)]^{2}},
$$

where $\boldsymbol{Q}=(\pi, \pi)$. The weight in the DOS at the Fermi level remains constant for small $U$; starts to decrease at $U \approx 6.35 t$ and smoothly goes to zero at $U=U_{\mathrm{c}}$. This is shown in Fig. 4, where $N(\mu)$, the density of states at the Fermi level, is plotted

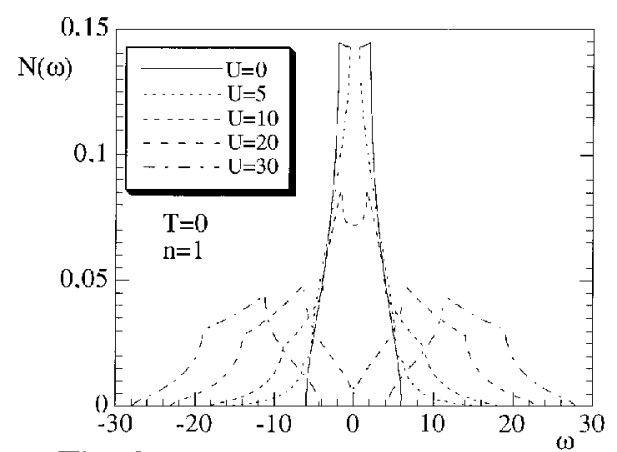

Fig. 3

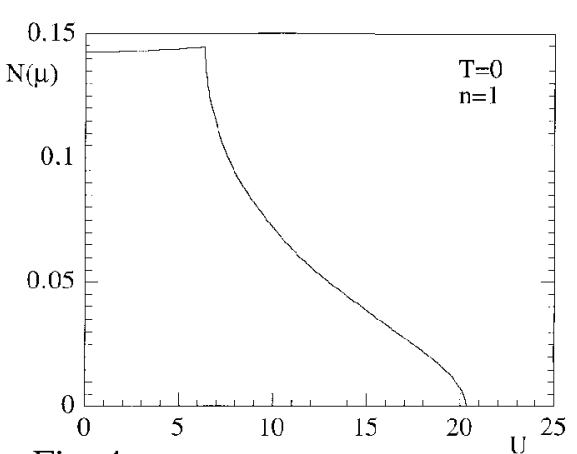

Fig. 4

Fig. 3. The electronic density of states is plotted as a function of the energy for half-filling, zero temperature and different values of $U$.

Fig. 4. The electronic density of states at the Fermi value is given against the potential strength for half-filling. 
versus $U$ at half-filling and zero temperature. This behavior is remarkably different from the $2 \mathrm{D}$ case, where the central peak at the Fermi level abruptly goes to zero at $U=U_{\mathrm{c}}$. From Eq. (3.2) we see that $U_{\mathrm{c}}$ is determined by the self-consistent equation

$$
U_{c}=12 t \sqrt{4 p-1}
$$

The self-consistent solution of this equation gives the result previously reported $U_{c} \approx 1.68 \mathrm{~W}$. Let us recall that this is the same value obtained in the $2 \mathrm{D}$ case [57], where $W=8 t$. Then, some characteristics of the $3 \mathrm{D}$ system can be obtained from the ones in the $2 \mathrm{D}$ system by scaling the band width. Let us notice that the value $U_{c}=4 W / 3$ has been obtained in Ref. [37] and that the Monte Carlo result on a $4^{3}$ lattice [30] demonstrates that the gap at $U=2 W / 3$ already exists. For $U>U_{\mathrm{c}}$ the distance between the two bands increases linearly with $U$. This is seen in Fig. 5 where the gap in the excitation spectrum is plotted versus $U$ for $n=1$ and $T=0$.

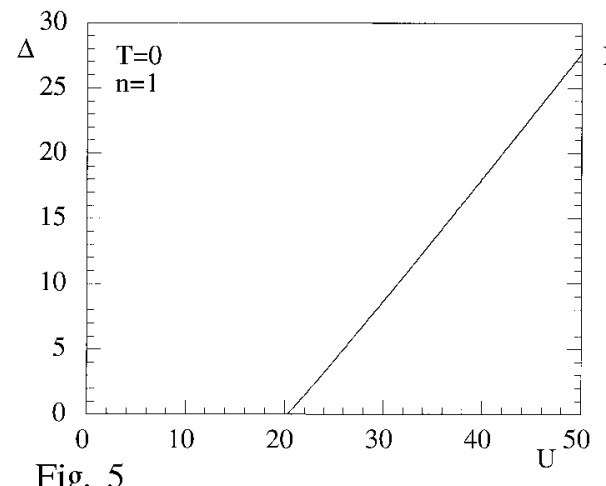

Fig. 5

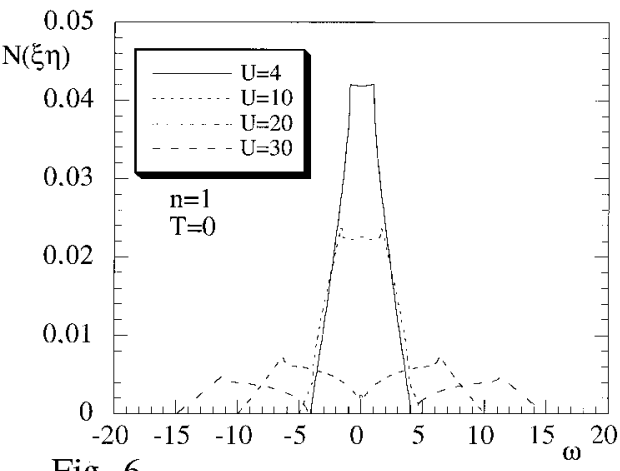

Fig. 6

Fig. 5. The gap in the excitation spectrum is plotted versus $U$ for $n=1$ and $T=0$.

Fig. 6. The cross term $2 N_{\xi \eta}(\omega)$ in the total electronic density of states is plotted as a function of the energy for half-filling, zero temperature and different values of $U$.

Our results for the density of states show that also for $U>U_{c}$, where the two subbands are separated, both fields $\xi$ and $\eta$ give contributions to the two bands. Although, the lower subband is essentially made up by the contribution of the " $\xi$-electron", there is always a contribution coming from the " $\eta$-electron". The vice versa is true for the upper subband. This is shown in Fig. 6 where the cross term $2 N_{\xi \eta}(\omega)$, appearing in the total density of states, is given as a function of the energy $\omega$ at zero temperature, half-filling and several values of $U$. We see that this term is different from zero also in the insulating phase (i.e. for $U>U_{\mathrm{c}}$ ). Only in the limit $U \rightarrow \infty$ the two fields do not interact. This result indicates that in the insulating phase the ground state of the system has some characteristic features. At half-filling the double occupancy $D=\left\langle n_{\uparrow} n_{\downarrow}\right\rangle=\frac{n}{2}-\left\langle\eta(i) \eta^{\dagger}(i)\right\rangle$ is given by [56]: 


$$
D=\frac{1}{4}\left(1-\frac{1}{2} U K_{3}\right),
$$

where $K_{3}$ is the quantity defined in Eq. (A.1). It is easy to see that in the limit $U \gg t$ the double occupancy decreases as $t^{2} / U^{2}$ and vanishes only for infinite $U$. For any finite value of $U$ there is always a small fraction of empty sites. Easy calculations and use of (A.10)-(A.11) show that at zero temperature we have

$$
\lim _{U \gg t} D=d \frac{t^{2}}{U^{2}}+\mathrm{O}\left(\frac{t^{4}}{U^{4}}\right)
$$

It is interesting to note that for large $U$ the double occupancy scales as the dimension $d$ of the system.

These results show that in the insulating phase the ground state has a structure different from the simple one where all sites are singly occupied; the competition between the itinerant and the local terms leads to a ground state characterized by a small fraction of empty and doubly occupied sites. Some questions arise:

(i) what is the structure of the ground state, and in particular there exists any order?

(ii) if an ordered state is established, can we individuate an order parameter describing the transition at $U_{\mathrm{c}}$ ?

For better understanding the structure of the ground state, we shall study the matrix element $\left\langle c_{\sigma}(j) c_{\sigma}^{\dagger}(i)\right\rangle$. This quantity represents the probability amplitude that an electron of spin $\sigma$ is created at site $i$ and an electron of spin $\sigma$ is destroyed at site $j$. However, this quantity gives only a limited information about the average occupation of the sites $i$ and $j$; there are four possible ways to realize the transition $j(\sigma) \rightarrow i(\sigma)$, and the quantity $\left\langle c_{\sigma}(j) c_{\sigma}^{\dagger}(i)\right\rangle$ cannot distinguish among them. By means of the decomposition $c_{\sigma}(i)=\xi_{\sigma}(i)+\eta_{\sigma}(i)$, the probability amplitude is written as the sum of four contributions

$$
\begin{aligned}
& \left\langle c_{\sigma}(j) c_{\sigma}^{\dagger}(i)\right\rangle \\
& \quad=\left\langle\xi_{\sigma}(j) \xi_{\sigma}^{\dagger}(i)\right\rangle+\left\langle\xi_{\sigma}(j) \eta_{\sigma}^{\dagger}(i)\right\rangle+\left\langle\eta_{\sigma}(j) \xi_{\sigma}^{\dagger}(i)\right\rangle+\left\langle\eta_{\sigma}(j) \eta_{\sigma}^{\dagger}(i)\right\rangle
\end{aligned}
$$

which correspond to different transitions (see Ref. [57]). A study of the probability amplitudes $\left\langle\psi(j) \psi^{\dagger}(i)\right\rangle$ will give detailed information about the structure of the ground state. We have computed these amplitudes for the specific case of half-filling and by considering transitions up to the third nearest neighbors.

In Fig. 7 the amplitudes $A^{\alpha}=\left\langle\xi^{\alpha}(i) \xi^{\dagger}(i)\right\rangle=\left\langle\eta^{\alpha}(i) \eta^{\dagger}(i)\right\rangle$ and $B^{\alpha}=$ $\left\langle\eta^{\alpha}(i) \xi^{\dagger}(i)\right\rangle=\left\langle\xi^{\alpha}(i) \eta^{\dagger}(i)\right\rangle$ are plotted versus $U$ at half-filling and zero temperature. The first amplitude vanishes at $U=U_{\mathrm{c}}$ and remains zero for all values of $U>U_{c}$, restricting the mobility of the electrons. The second quantity does not satisfy this property; owing to this contribution, we have that for $U>U_{c}$ the hopping of electrons from site $i$ to the nearest neighbor is not forbidden, although restricted by the fact that $A^{\alpha}=0$. The probability amplitudes of hopping to the second and third nearest neighbors have been also calculated. The picture emerging from this 


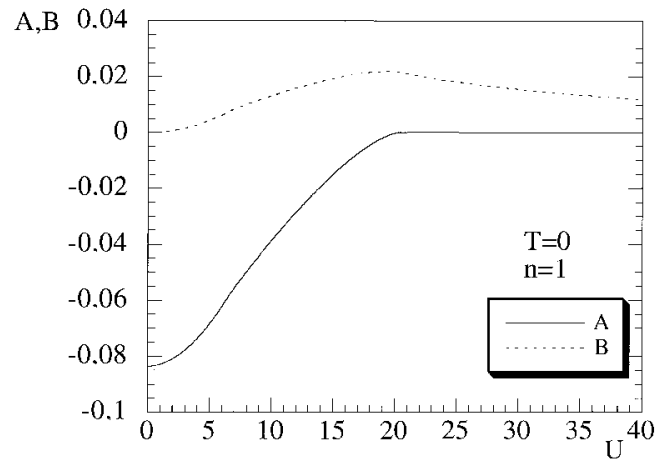

Fig. 7. The first nearest-neighboring hopping amplitudes $A^{\alpha}=\left\langle\xi^{\alpha}(i) \xi^{\dagger}(i)\right\rangle$ and $B^{\alpha}=\left\langle\xi^{\alpha}(i) \eta^{\dagger}(i)\right\rangle$ are plotted versus the potential strength $U$ for half-filling and zero temperature.

study confirms the one found [57] for the 2D Hubbard model. The ground state in the insulating phase is characterized by a local antiferromagnetic order. Due to the fact that there are empty and doubly occupied sites, the electrons have some mobility, but there are strong constraints on this mobility, such that the local antiferromagnetic order is not destroyed. The matrix elements $\left\langle\xi_{\sigma}\left(j_{\text {odd }}\right) \xi_{\sigma}^{\dagger}(i)\right\rangle$ and $\left\langle\eta_{\sigma}\left(j_{\text {odd }}\right) \eta_{\sigma}^{\dagger}(i)\right\rangle$ might be considered as the quantities which control the order in the insulating state.

\section{Conclusions}

The three-dimensional Hubbard model has been studied by means of the composite operator method. By considering as basic set of field operators the Hubbard operators $\xi$ and $\eta$, which describe singly and doubly occupied lattice sites, respectively, the single-particle Green function has been computed by using the equations of motion in the polar approximation. The paper is dedicated to the study of the Mott-Hubbard transition, therefore, the special case of half-filling and zero temperature has been considered. Once the self-consistent equations have been solved for the paramagnetic case, the density of states has been computed for a large range of $U / t$ values. Analytical calculations show the existence of a critical value $U_{\mathrm{c}}$, defined by a self-consistent equation and mainly controlled by intersite correlations, which separates the metallic and insulating phases. For $U>U_{\mathrm{c}}$ a gap opens and the density of states splits into two separated structures. An interesting result is obtained when we separately calculate the contribution of the fields $\xi$ and $\eta$ to the global density of states. Our calculations show that even for $U>U_{\mathrm{c}}$, where the lower and upper bands are well separated, the two contributions coming from $\xi$ and $\eta$ do not separate. The ground state in the insulating phase contains always a small fraction of empty and doubly occupied sites.

This result is confirmed by the calculation of the matrix element $\left\langle c_{\sigma}(j) c_{\sigma}^{\dagger}(i)\right\rangle$, which gives the probability amplitude of hopping from the site $j$ to the site $i$. We 
find that when $j$ is an odd nearest neighboring site of $i$, this quantity is not zero for $U>U_{\mathrm{c}}$ and vanishes only for infinite $U$. However, when we split $c=\xi+\eta$ and analyze $\left\langle c_{\sigma}\left(j_{\text {odd }}\right) c_{\sigma}^{\dagger}(i)\right\rangle$ in components, we find that for $U>U_{\mathrm{c}}$ only the matrix elements $\left\langle\xi_{\sigma}\left(j_{\text {odd }}\right) \eta_{\sigma}^{\dagger}(i)\right\rangle$ and $\left\langle\eta_{\sigma}\left(j_{\text {odd }}\right) \xi_{\sigma}^{\dagger}(i)\right\rangle$ survive. The probability amplitudes $\left\langle\xi_{\sigma}\left(j_{\text {odd }}\right) \xi_{\sigma}^{\dagger}(i)\right\rangle$ and $\left\langle\eta_{\sigma}\left(j_{\text {odd }}\right) \eta_{\sigma}^{\dagger}(i)\right\rangle$ vanish at $U=U_{\mathrm{c}}$ and remain zero for all $U>U_{\mathrm{c}}$. On the other hand, the matrix element $\left\langle c_{\sigma}\left(j_{\text {even }}\right) c_{\sigma}^{\dagger}(i)\right\rangle$ is always zero for any value of $U$; the two contributions $\left\langle\xi_{\sigma}\left(j_{\text {even }}\right) \xi_{\sigma}^{\dagger}(i)\right\rangle$ and $\left\langle\eta_{\sigma}\left(j_{\text {even }}\right) \eta_{\sigma}^{\dagger}(i)\right\rangle$ compensating each other.

Summarizing, our calculations suggest that the ground state of the Mott insulator has the following characteristics:

(i) a small fraction of sites are empty or doubly occupied; the number of these sites depend on the value of $U / t$ and tends to zero only in the limit $U \rightarrow \infty$;

(ii) a local antiferromagnetic order is established;

(iii) the electrons keep some mobility, but this mobility must be compatible with the local antiferromagnetic order;

(iv) the matrix elements $\left\langle\xi_{\sigma}\left(j_{\text {odd }}\right) \xi_{\sigma}^{\dagger}(i)\right\rangle$ and $\left\langle\eta_{\sigma}\left(j_{\text {odd }}\right) \eta_{\sigma}^{\dagger}(i)\right\rangle$ might be considered as the quantities which control the order in the insulating phase.

\section{Acknowledgments}

V.T. wishes to thank the members of the Dipartimento di Fisica "E.R. Caianiello", Università degli Studi di Salerno, Italy, especially Prof. F. Mancini for their kind hospitality. The authors wish to thank Dr. Adolfo Avella for the many useful discussions and his careful reading of the manuscript.

\section{Appendix}

The quantities $K_{i}(i=1,7)$, introduced in Sec. 2 , are defined as

$$
\begin{aligned}
& K_{1} \equiv \frac{1}{8 k_{\mathrm{B}} T} \frac{\Omega}{(2 \pi)^{d}} \int_{\Omega_{\mathrm{B}}} \mathrm{d}^{d} k\left[t_{10}^{2}(k)+t_{20}^{2}(k)-2\right], \\
& K_{2} \equiv \frac{1}{8 k_{\mathrm{B}} T} \frac{\Omega}{(2 \pi)^{d}} \int_{\Omega_{\mathrm{B}}} \mathrm{d}^{d} k \alpha(k) \frac{t_{10}^{2}(k)-t_{20}^{2}(k)}{Q_{0}(k)}, \\
& K_{3} \equiv \frac{\Omega}{2(2 \pi)^{d}} \int_{\Omega_{\mathrm{B}}} \mathrm{d}^{d} k \frac{t_{10}(\boldsymbol{k})-t_{20}(\boldsymbol{k})}{Q_{0}(\boldsymbol{k})}, \\
& K_{4} \equiv \frac{1}{8 k_{\mathrm{B}} T} \frac{\Omega}{(2 \pi)^{d}} \int_{\Omega_{\mathrm{B}}} \mathrm{d}^{d} k \alpha^{2}(k) \frac{2-t_{10}^{2}(k)-t_{20}^{2}(k)}{Q_{0}^{2}(k)}, \\
& K_{5} \equiv \frac{\Omega}{2(2 \pi)^{d}} \int_{\Omega_{\mathrm{B}}} \mathrm{d}^{d} k \alpha^{2}(\boldsymbol{k}) \frac{t_{10}(\boldsymbol{k})-t_{20}(k)}{Q_{0}(\boldsymbol{k})},
\end{aligned}
$$




$$
\begin{aligned}
& K_{6} \equiv \frac{\Omega}{2(2 \pi)^{d}} \int_{\Omega_{\mathrm{B}}} \mathrm{d}^{d} k \alpha^{2}(\boldsymbol{k}) \frac{t_{10}(\boldsymbol{k})-t_{20}(\boldsymbol{k})}{Q_{0}^{3}(\boldsymbol{k})}, \\
& K_{7} \equiv \frac{\Omega}{2(2 \pi)^{d}} \int_{\Omega_{\mathrm{B}}} \mathrm{d}^{d} k \alpha(\boldsymbol{k})\left[t_{10}(\boldsymbol{k})+t_{20}(\boldsymbol{k})\right],
\end{aligned}
$$

where

$$
\begin{aligned}
& t_{10}(k)=\tanh \left(\frac{E_{1}^{(0)}(k)}{2 k_{\mathrm{B}} T}\right), \quad t_{20}(k)=\tanh \left(\frac{E_{2}^{(0)}(k)}{2 k_{\mathrm{B}} T}\right), \\
& E_{1}^{(0)}(k)=R_{0}(k)+Q_{0}(k), \quad E_{2}^{(0)}(k)=R_{0}(k)-Q_{0}(k), \\
& R_{0}(k)=-4 d t p \alpha(k), \quad Q_{0}(k)=\sqrt{\frac{U^{2}}{4}+4 d^{2} t^{2}(2 p-1)^{2} \alpha^{2}(k)} .
\end{aligned}
$$

When the hopping $t_{i j}$ is restricted to first nearest neighbors, all integrations in momentum space can be reduced to one-dimensional integration. Let us consider the integral

$$
\frac{\Omega}{(2 \pi)^{d}} \int_{\Omega_{\mathrm{B}}} \mathrm{d}^{d} k F[\alpha(k)]
$$

where $F[\alpha(\boldsymbol{k})]$ is a generic function of $\alpha(\boldsymbol{k})$, defined by (2.6). It is straightforward to derive the following formula:

$$
\frac{\Omega}{(2 \pi)^{d}} \int_{\Omega_{\mathrm{B}}} \mathrm{d}^{d} k F[\alpha(k)]=\int_{-1}^{1} \mathrm{~d} x F[x] w(x),
$$

where

$$
w(x)=\frac{a^{d}}{(2 \pi)^{d}} \int_{\Omega_{\mathrm{B}}} \mathrm{d}^{d} k \delta[x-\alpha(\boldsymbol{k})] .
$$

The expression of the function $w(x)$ depends on the spatial dimension. Straightforward calculations give the following results:

one dimension

$$
w(x)=\frac{1}{\pi} \frac{1}{\sqrt{1-x^{2}}},
$$

two dimensions

$$
w(x)=\frac{2}{\pi^{2}} K\left(\sqrt{1-x^{2}}\right),
$$

three dimensions [58]

$$
\begin{aligned}
w(x) & =\frac{24}{(2 \pi)^{3}} \int_{3 x-2}^{1} \frac{\mathrm{d} y}{\sqrt{1-y^{2}}} K^{\prime}\left(\frac{3 x-y}{2}\right) \Theta\left(x-\frac{1}{3}\right) \\
& +\frac{24}{(2 \pi)^{3}} \int_{-1}^{1} \frac{\mathrm{d} y}{\sqrt{1-y^{2}}} K^{\prime}\left(\frac{3 x-y}{2}\right) \Theta\left(\frac{1}{3}-x\right),
\end{aligned}
$$

where $K(x)$ is the complete elliptic integral of the first kind and $K^{\prime}(x)=K\left(\sqrt{1-x^{2}}\right)$. 
We note that the formula (A.9) is valid for $x \geq 0$; but it is easy to see that $w(-x)=w(x)$. It is possible to see that (A.7)-(A.9) satisfy the relations

$$
\begin{aligned}
& \int_{-1}^{1} \mathrm{~d} x w(x)=1 \\
& \int_{-1}^{1} \mathrm{~d} x x^{2} w(x)=\frac{1}{2 d}
\end{aligned}
$$

\section{References}

[1] B.H. Brandow, Adv. Phys. 26, 651 (1977).

[2] J.H. de Boer, E.J.W. Verwey, Proc. Phys. Soc. 49, 59 (1937).

[3] N.F. Mott, Proc. Phys. Soc. A 62, 416 (1949); Proc. Metal. Phys. 3, 76 (1952); Can. J. Phys. 34, 1356 (1956); Suppl. to Nuovo Cimento 7, 312 (1958); Philos. Mag. 6, 28 (1961).

[4] M.C. Gutzwiller, Phys. Rev. Lett. 10, 159 (1963); J. Hubbard, Proc. R. Soc. Lond. A 276, 238 (1964)); J. Kanamori, Prog. Theor. Phys. 30, 275 (1963).

[5] N.F. Mott, Metal-Insulator Transitions, Taylor and Francis, London 1990.

[6] F. Gebhard, The Mott Metal-Insulator Transition, Springer-Verlag, Berlin 1997.

[7] M. Imada, F. Fujimori, Y. Tokura, Rev. Mod. Phys. 70, 1039 (1998).

[8] H.A. Bethe, Z. Phys. 71, 205 (1931).

[9] E.H. Lieb, F.Y. Wu, Phys. Rev. Lett. 20, 1445 (1968).

[10] N.F. Mott, Rev. Mod. Phys. 40, 677 (1968).

[11] W.F. Brinkman, T.M. Rice, Phys. Rev. B 2, 4302 (1970).

[12] P.W. Anderson, Solid State Phys. 14, 99 (1963).

[13] Y. Nagaoka, Phys. Rev. 147, 392 (1966).

[14] P.W. Anderson, Science 235, 1196 (1987).

[15] S.E. Barnes, J. Phys. F 6, 1375 (1976); ibid. 7, 2637 (1977); G. Kotliar, A.E. Ruckenstein, Phys. Rev. Lett.57, 1362 (1986); L. Lilly, A. Maramatsu, W. Hanke, Phys. Rev. Lett. 65, 1379 (1990); R. Frésard, M. Dzierzawa, P. Wölfe, Europhys. Lett. 65, $1379(1990)$.

[16] W. Metzner, D. Vollhardt, Phys. Rev. Lett. 62, 324 (1989).

[17] A. Georges, G. Kotliar, Phys. Rev. B 45, 6479 (1992).

[18] K.W. Becker, W. Brenig, P. Fulde, Z. Phys. B 81, 165 (1990); A.J. Fedro, Yu Zhou, T.C. Leung, B.N. Harmon, S.K. Sinha, Phys. Rev. B 46, 14785 (1992); P. Fulde, Electron Correlations in Molecules and Solids, Springer, Berlin 1993.

[19] (a) F. Mancini, S. Marra, H. Matsumoto, Physica C 244, 49 (1995); ibid. 250, 184 (1995); ibid. 252, 361 (1995); (b) A. Avella, F. Mancini, D. Villani, L. Siurakshina, V. Yushankhai, Int. J. Mod. Phys. B 12, 81 (1998); (c) F. Mancini, A. Avella, Condens. Matter Phys. 1, 11 (1998); (d) A. Avella, F. Mancini, R. Münzner, Phys. Rev. B 63, 245117 (2001). 
[20] F. Mancini, H. Matsumoto, D. Villani, Phys. Rev. B 57, 6145 (1998); A. Avella, F. Mancini, D. Villani, Phys. Lett. A 240, 235 (1998); A. Avella, F. Mancini, D. Villani, Solid State Commun. 108, 723 (1998); A. Avella, F. Mancini, D. Villani, Physica B 259-261, 732 (1999); F. Mancini, V. Turkowski, Physica B 284-8, 1575 (2000); A. Avella, F. Mancini, H. Matsumoto, D. Villani, Eur. Phys. J. B 20, 303 (2001)

[21] A. Fujimori, I. Hase, H. Namatame, Y. Fujishima, Y. Tokura, H. Eisaki, S. Uchida, K. Takegahara, F.M.F. de Groot, Phys. Rev. Lett. 69, 1796 (1992).

[22] I.H. Inoue, I. Hase, Y. Aiura, A. Fujimori, Y. Haruyama, T. Maruyama, Y. Nishihara, Phys. Rev. Lett. 74, 2539 (1995).

[23] K. Morikawa, T. Mizokawa, K. Kobayashi, A. Fujimori, H. Eisaki, S. Uchida, F. Iga, Y. Nishihara, Phys. Rev. B 52, 13711 (1995).

[24] F.C. Chou, D.C. Johnston, Phys. Rev. B 54, 572 (1996).

[25] Y. Taguchi, Y. Tokura, T. Arima, F. Inaba, Phys. Rev. B 48, 511 (1993).

[26] Y. Tokura, Y. Taguchi, Y. Okada, Y. Fujishima, T. Arima, K. Kumagai, Y. Iye, Phys. Rev. Lett. 70, 2126 (1993).

[27] M.F. Hundley, J.D. Thompson, S.-W. Cheong, Z. Fisk, J.E. Schirber, Phys. Rev. $B$ 41, 4062 (1990).

[28] J. Ryder, P.A. Midgley, R. Exley, R.J. Beynon, D.L. Yates, L. Afalfiz, J.A. Wilson, Physica C 173, 9 (1991).

[29] Jing Rong-ying, Zhao Guo-meng, Qi Ze Ran, Solid State Commun. 67, 415 (1988).

[30] M. Ulmke, R.T. Scalettar, A. Nazarenko, E. Dagotto, Phys. Rev. B 55, 16523 (1996).

[31] A. Fujimori, I. Hase, M. Nakamura, H. Namatame, Y. Fujishima, Y. Tokura, M. Abbate, F.M.F. de Groot, M.T. Czyzyk, J.C. Fuggle, O. Strebel, F. Lopez, M. Domke, G. Kaindl, Phys. Rev. B 46, 9841 (1992).

[32] M. Jarrell, T. Pruschke, Z. Phys. B 90, 187 (1993).

[33] T. Pruschke, M. Jarrell, J.K. Freericks, Adv. Phys. 44, 187 (1995).

[34] H. Schweitzer, G. Czycholl, Z. Phys. B 83, 93 (1991).

[35] G. Bulk, R.J. Jelitto, Phys. Rev. B 41, 413 (1990).

[36] J.M. Dixon, J.A. Tuszyński, Int. J. Mod. Phys. B 12, 2565 (1998).

[37] M. Potthoff, W. Nolting, Eur. Phys. J. B 8, 555 (1999).

[38] R. Arita, Sh. Onoda, K. Kuroki, H. Aoki, http://arxiv.org/abs/cond-mat/0002441.

[39] J.E. Hirsch, Phys. Rev. B 35, 1851 (1987).

[40] A.M. Daré, G. Albinet, http://arxiv.org/abs/cond-mat/9909243.

[41] Y. Otsuka, Y. Hatsugai, http://arxiv.org/abs/cond-mat/0005270.

[42] R.T. Scalettar, D.J. Scalapino , R.L. Sugar, D. Toussaint, Phys. Rev. B 39, 4711 (1989).

[43] R. Staudt, M. Dzierzawa, A. Muramatsu, http://arxiv.org/abs/cond-mat/0007042, to be published in Eur. Phys. J. B (2000).

[44] Y.H. Szczech, M.A. Tusch, D.E. Logan, Phys. Rev. Lett. B 74, 2804 (1995). 
[45] M.A. Tusch, Y.H. Szczech, D.E. Logan, Phys. Rev. B 53, 5505 (1996).

[46] A.N. Tahvildar-Zadeh, J.K. Freericks, M. Jarrell, http://arxiv.org/abs/cond-mat/9604039.

[47] K.K. Pan, Yu. L. Wang, Phys. Rev. B 55, 2981 (1997).

[48] Y. Kakehashi, P. Fulde, Phys. Rev. B 32, 1595 (1985).

[49] Y. Kakehashi, J. Samson, Phys. Rev. B 33, 298 (1986).

[50] Y. Kakehashi, H. Hasegawa, Phys. Rev. B 36, 4066 (1987).

[51] Y. Kakehashi, H. Hasegawa, Phys. Rev. B 37, 7777 (1988).

[52] W. Metzner, D. Vollhardt, Phys. Rev. B 39, 4462 (1989).

[53] G. Migliorini, A.N. Berker, http://arxiv.org/abs/cond- mat/9811233.

[54] F. Mancini, A. Avella, http://arxiv.org/abs/cond-mat/0006377.

[55] A. Avella, F. Mancini, M. del Mar Sanchez-Lopez, Europhys. Lett. 44, 328 (1998); http://arxiv.org/abs/cond-mat/0008016.

[56] F. Mancini, M. Marinaro, H. Matsumoto, Int. J. Mod. Phys. B 10, 1717 (1996).

[57] F. Mancini, Europhys. Lett. 50, 229 (2000).

[58] R.J. Jelitto, J. Phys. Chem. Solids 30, 609 (1969). 